\title{
Trump Tweets
}

\author{
Cynthia Whissell \\ Laurentian University, Sudbury, ON
}

*Corresponding Author: Cynthia Whissell, Laurentian University, Sudbury, ON, Canada. Email: Cwhissell@Laurentian.Ca

\begin{abstract}
President Trump's 2017 tweets $(N=2,602)$ were studied in terms of their pleasantness (as measured by the Dictionary of Affect; Whissell, 2009), their popularity (defined in terms of retweets and likes), message characteristics (e.g., message length), and message content (e.g., whether the message included mentions of Melania, CNN, Hillary Clinton, or Democrats). Pleasantness and popularity were significantly predicted $(p<.001)$ on the basis of message characteristics and content variables. Table 3 highlights the presence of two types of message: pleasant/unpopular tweets and unpleasant/popular ones. On the basis of their characteristics and contents, the first type of tweet was labeled as celebratory/ congratulatory and the second as antagonistic/accusatory. Antagonistic/accusatory tweets tended to mention Trump's political opponents (e.g., Clinton, Democrats, CNN) and to be posted between 7:00 pm and midnight. Trump's tweets were mildly pleasant in tone, but not as pleasant as tweets posted by President Obama in 2015-2016.
\end{abstract}

Keywords: Trump, Twitter, emotion, popularity

\section{INTRODUCTION}

During 2017, Donald J. Trump posted an average of more than seven tweets a day. In the immediately previous years, Trump had employed tweets as a campaign strategy in his run for the U.S. presidency (Enli, 2017; Lee \& Lim, 2016; Ott, 2017; Wang, Luo, Niemi, Li,\& Hu, 2016), and he continued to tweet at a high rate once in office. Twitter is a relatively new communication technology. Presidents Obama and Trump both tweeted, but Trump tweeted at a much higher rate. As reported in sources described in the Methodology section below, Obama tweeted 321 times in his last 19 months in office, while Trump tweeted 2,602 times in his first year.

This research addresses the pleasantness/unpleasantness of the language in Trump's 2017 tweets. It questions whether this pleasantness/unpleasantness is related to tweet characteristics (such as their length in number of words, their inclusion of a hash tag, or their status as a reply) or to information included within tweet text (such as an American flag emoji, an exclamation mark, or a mention of $\mathrm{CNN}$ ). These variables are employed to predict pleasantness, and pleasantness in turn is employed with the other predictors to identify popular tweets that were retweeted at a high rate.

\subsection{Measuring the Pleasantness/Unpleasantness of Tweets}

Sentiment analysis is an approach that examines the emotion conveyed - in tweets, in this case-by looking at the words in them. Some forms of sentiment analysis depend on lists of words indicative of various emotional states; they evaluate the occurrence of these words in tweets and infer emotionality from occurrence, sometimes in very sophisticated ways (e.g., Mohammad, Kiritchenko, \& Zhu, 2013; Saif, Fernandez, He, \& Alani, 2016). The approach employed in this research depends instead on the rated emotional connotations of many thousands of words, including some very common ones (Whissell, 2009). Ratings contained in the Dictionary of Affect in Language (Whissell, 2009) ${ }^{1}$ were provided by multiple raters. They had been obtained, several years before the current research, in a totally independent setting where participants rated context-free words on their pleasantness. The scale employed in this research is a simple linear transformation of the original scale. The tool has been applied to many different types of data: from Shakespeare (Whissell, 2010a) to President Clinton's communications (Whissell, 2010b).

\footnotetext{
${ }^{1}$ Referred to in the rest of this article as the Dictionary, or the Dictionary of Affect
} 
A benefit of this approach to measuring pleasantness is that it scores most of the words in a text in order to provide a stable and accurate overall assessment of its emotionality. A limitation is that it provides scores for entire tweets, but does not address segments within them. Given the extremely compact nature of tweets, however, this is likely not a serious limitation. One expects considerable emotional variability within a novel, for example, but not within 140 characters.

In order to calculate the pleasantness/unpleasantness of Trump's tweets, each word in each tweet was matched to the Dictionary, and matched words contributed to the pleasantness or unpleasantness of the message. Pleasantness scores have a 120 point range; samples of everyday English tend to have averages close to 50. Below are two Trump 2017 examples. Tweet \#1 is an extremely pleasant tweet from November 2, and Tweet \#2 an extremely unpleasant one from June 13. The number following each word below is its pleasantness score from the Dictionary of Affect; in a few cases there was no matching word, and therefore no score was available.

Tweet \#1. Happy (119) birthday (99) to (46) @ garyplayer (no score), a (59) truly (107) great (97) champion (59)! [pleasantness average: 98 ]

Tweet \#2. The (44) Fake (11) News (46) Media (29) has (46) never (19) been (33) so (44) wrong (14) or (29) so (44) dirty (20). Purposely (no score) incorrect (no score) stories (67) and (39) phony (14) sources (53) to (46) meet (66) their (48) agenda (no score) of (37) hate (11).

Sad (21)! [Pleasantness average: 36]

The tweets' pleasantness averages (98 versus 36 ) clearly reflect differences in message emotionality. The first tweet is considerably more pleasant and the second more unpleasant. The tweets include words near the extreme ranges of the Dictionary (e.g., happy, with a score of 119; and hate, with a score of 11), as well as words closer to the average (e.g., their, with a score of 48 ; and sources, with a score of 53).

\subsection{Questions and Predictions of the Research}

It has been suggested by more than one researcher that tweets in general and Trump's tweets in particular are emotionally negative (Lee \& Lim, 2016; Ott, 2017). This would lead to an expectation of a pleasantness average of below 50, the level typical of everyday English, for Trump's tweets; they were thus predicted to fall closer to the second of the two examples above than to the first. Other researchers have noted that tweets addressing topics such as CNN, fake news, Hillary Clinton, or the Democrats tended to be more appealing to Trump followers (Wang et al., 2016). The prediction from this research was that such generally accusatory tweets would be more popular and also less pleasant than the remaining tweets. On the other hand, it seemed likely that Trump's tweets including an American flag emoji or mentioning Melania (the First Lady), Mar-a-Lago (Trump's vacation home in Florida),or Fox (a right-leaning network which tends to support the president) would be more pleasant than other tweets.

As a collection, tweet characteristics and tweet text content measures are expected to be predictive of popularity, since this is reflected in retweeting; and also of pleasantness. On the basis of the emphasis placed on negative emotions in tweets, it was predicted that the messages retweeted the most would be the more unpleasant ones. The unpleasant example above (\#2) was retweeted 25,099 times, while the pleasant one (\#1) was retweeted only 5,181 times.

\section{Methodology}

Trump's tweets were obtained on April 26, 2018, from a web site overseen by Brandon Brown (2018). Three tweets with dates in 2018 were discarded from the set, leaving a total of 2,602 tweets from 2017. These represent a tweeting rate of more than seven tweets per day. Associated with each tweet was information as to its status as a reply or a retweet, the number of times it had been tagged with a like, and the number of times it had been retweeted. String-matching techniques were employed to determine whether reach tweet included a mention (denoted by the symbol @) or a hashtag (denoted by the symbol \#).

The text of each tweet was examined by a program written by the author using IBM-SPSS statistical software, by which string variables that began with letters were identified as words. It counted the number of words in each tweet. The count excluded hashtags, mention symbols, and emojis because these did not begin with letters of the alphabet. Each word was matched to the Dictionary of Affect which provided pleasantness/unpleasantness ratings for a majority of the words. 
As illustrated earlier, the pleasantness value of individual words was averaged for each tweet, to provide a tweet pleasantness mean. Pleasantness means were available for 2,556 (98\%) tweets; 46 tweets did not include any emotionally matched words, probably because they were very limited in their vocabulary. Unscored tweets (average length $=3.48$ words) were significantly ( $t$-test, $p<.001)$ and considerably $(d>2)$ shorter than scored tweets (21.81 words). For example, one unscored tweet was posted on April 24; in it Trump was congratulating an astronaut. The tweet only included the hashtag \#CongratsPeggy, an exclamation mark, and an http address. The matching rate of the Dictionary for normal everyday English is 90\% (Whissell, 2009). The matching rate for Trump's tweet's was 77\%, which makes sense in terms of the condensed language of tweets and the fact that they frequently included proper nouns such as names of individuals or agencies.

Tweets were examined for the presence/absence of 10 specific word categories (Table 1) which were scored as 0 or 1 . For example, the category Democrats was scored as positive if the tweet included any of the strings Dem, Dems, Democrat, or Democrats. The category Hillary was considered as present if either Hillary or Clinton appeared in the tweet. Similarly, the category $C N N$ was considered present if either $C N N$ or $C n n$ appeared in the message, and a similar criterion was employed for Fox (FOX or Fox) and fake news (Fake News or fake news, in sequence). If Melania or Mar-a-Lago appeared in a tweet, these variables too were scored as 1 rather than 0 . The presence of the word America earned a 1 for the eponymous category, as did the presence of an American flag emoji or an exclamation mark for their categories. Six tweet characteristics (number of words, status as a reply, status as a retweet, inclusion of a hashtag symbol, inclusion of a mention symbol, and time of year) were also employed in prediction. Total words were counted as described above, and time of year was represented by a variable ranging from 1 to 2,602 (earliest to latest).

To help test the first hypothesis, which refers to the expected unpleasantness of Trump's tweets, a sample of tweets was obtained from Barack Obama who had his own personal Twitter account, @POTUS, beginning in May 2015. Obama's Tweets posted in 2015 and 2016-321 in all-were made available in a White House archive shortly before the end of his second term in office (The Internet Archive, n.d.). The Dictionary matching rate for Obama tweets was 78\%. Although the Obama and Trump tweets were sampled from adjacent years (2015-2017), differences in tweet content and pleasantness may be due in part to differences in the situation of each tweeter (e.g., outgoing versus incoming president) and in part to extreme differences in the frequency of tweeting (roughly once every other day for Obama and seven times a day for Trump).

Table1. Sixteen Descriptors of Trump's 2017 Tweets and Their Role as Predictors of Pleasantness

\begin{tabular}{|l|c|c|c|}
\hline & $\begin{array}{c}\text { Correlation with } \\
\text { pleasantness }\end{array}$ & $\begin{array}{c}\text { Beta predicting } \\
\text { pleasantness }\end{array}$ & \% tweets or mean \\
\hline Tweet characteristics & & & $.092^{*}$ \\
\hline Time of year & .024 & $-.256^{*}$ & 1301.5 \\
\hline Number of words & $-.266^{*}$ & $-.097^{*}$ & 11.49 \\
\hline Is a retweet & -.025 & $-.041^{*}$ & $1.7 \%$ \\
\hline Is a reply & -.033 & $.045^{*}$ & $17.3 \%$ \\
\hline Includes a hashtag & $.145^{*}$ & $.049^{*}$ & $28.3 \%$ \\
\hline Includes a mention & $.057^{*}$ & & $12.1 \%$ \\
\hline Tweet content & & $.081^{*}$ & $4.5 \%$ \\
\hline Mentions America & $.112^{*}$ & $.072^{*}$ & $3.1 \%$ \\
\hline Includes American flag emoji & $.145^{*}$ & $-.053^{*}$ & $7.3 \%$ \\
\hline Mentions Hillary & $-.094^{*}$ & $-.156^{*}$ & $4.5 \%$ \\
\hline Mentions Democrats & $-.201^{*}$ & $-.109^{*}$ & $2.8 \%$ \\
\hline Mentions fake news & $-.158^{*}$ & $-.044^{*}$ & $1.4 \%$ \\
\hline Mentions Fox & -.034 & $-.052^{*}$ & $55.8 \%$ \\
\hline Mentions CNN & $-.083^{*}$ & $.115^{*}$ & $1.3 \%$ \\
\hline Includes exclamation mark & $.103^{*}$ & $.058^{*}$ & $.3 \%$ \\
\hline Mentions Melania & $.082^{*}$ & .027 & \\
\hline Mentions Mar-a-Lago & .020 & & \\
\hline
\end{tabular}

Note: ${ }^{1} N=2556$ messages with pleasantness scores, all predictors entered simultaneously, $R=.424 .{ }^{*} p<.05$ 


\subsection{Corrective Action}

According to the central limit theorem (discussed in most introductory social science statistics texts such as Gravetter, Wallnau, \& Forzano, 2018, p. 175), any measure depending on a mean is sensitive to sample size, with extreme means being more likely to occur with smaller samples. To correct for sample size, the pleasantness of each of Trump's tweets was represented by its distance (from the mean of the set) in terms of standard errors. With this correction, an extremely pleasant or an extremely unpleasant short tweet was treated as being less extreme than a longer tweet with a similar score. Because most tweets were of roughly similar length, the correlation $(r)$ between raw pleasantness and corrected pleasantness was .96 ( $p<.0001)$, suggesting that no violence had been done to the variable by transformation. Regression diagnostics in the analyses performed below presented no problems with the corrected measure as the criterion. The corrected pleasantness measure was employed in every analysis except for the one evaluating the first hypothesis. The number of retweets was strongly correlated to the number of likes $(\rho=.88, p<.0001)$, although the distributions for both variables were extremely positively skewed. Log (base 10) transformations failed to normalize the variables, so it was decided to employ a median split when analyzing them. A median split for retweets, for example, puts each message in one of two categories: in the top 50\% of retweeted tweets or in the bottom $50 \%$ of retweeted tweets.

\subsection{Limitations of the Measurements}

The measurement techniques employed in this research (e.g., the Dictionary of Affect, content mentions of Hillary Clinton, and other categories) were cleanly and clearly operationalized so that they could be calculated within a computer program, but they are not error-free. The Dictionary was developed on the basis of context-free scoring and context can alter meaning. The word funny changes meaning profoundly in the phrase not funny which was employed by Trump in a mildly unpleasant tweet (mean $=48.75$ ) on January 15. As well, the string matching employed to capture categories of mention might have missed the occasional reference. For example, if Trump referred to opponent instead of Hillary or Clinton, the tweet would not have been scored as mentioning Hillary, as happened with a January 11 tweet where Trump refers to crooked opponents. That being said, the measures employed in this research led to many significant results, and when a significant proportion of variability is captured by a measure which then demonstrates meaningful interrelationships with other variables, that measure must be deemed to be success. Future research might address a tighter definition of content variables.

Another limitation rests with the source of the tweets. There is no guarantee that each and every tweet originated from a presidential inspiration, or that the tweets were posted personally by the president concerned. In the media culture of Twitter, source addresses are known, but the poster and follower are both anonymous and may even be robotic (Borowitz, 2018; Ott, 2017).

\section{RESULTS}

There were 55,915 words in the data set, and average tweet length was 21.5 words. For descriptive purposes, words appearing at least 100 times in the full set were considered frequent. Frequent words included several first person pronouns ( $I, m e, m y$, we, and $u s$ ) and one second person pronoun (you). Recurrent negation was indicated by the presence of no and not at high rates (128 and 211 times), and a focus on the media by the frequent mentions of fake, news, and media. An appeal to Trump's voting base was suggested by the common occurrence of people, America, American, country, tax, and jobs, and Trump regularly included his name and office (Trump, president) in his tweets, although the office could be referring to that of visiting dignitaries. Trump's favorite adjective (great) appeared 501 times and the qualifier very 179 times. Additional words with frequencies greater than 100 included thank and big and several highly common English words such as the and of. The ratio of types (distinct words, $N=6,579$ ) to tokens (total words, $N=55,195$ ) was .12 , indicating that individual types (words) were repeated 8.39 times, on the average.

\subsection{Pleasantness}

The raw pleasantness average for Trump's tweets was 51.27. This number was significantly higher than the everyday English comparative average of $50(t=7.05, p<.001, d=.14)$. Rather than being unpleasant or below 50, as predicted, the tweets were mildly pleasant overall. Forty-eight percent of the tweets did have pleasantness scores below 50 , but $52 \%$ of them had higher scores. Obama's tweets 
had a mean pleasantness of 53.28 and were significantly more pleasant $(t=4.57, p<.001, d=.22)$ than the Trump tweets. Both Presidents tweeted using pleasant language, but Obama's language was more pleasant than Trump's.

The percentage of tweets including each of the categorical variables and the mean for time of year and number of words are reported in the last column of Table 1. Exclamation marks, hashtags, and mentions were all commonly included in tweets while references to CNN, Melania, and Mar-a-Lago were relatively rare. Trump retweeted frequently-more than one out of every 10 messages was a retweet - and he often included hashtags and mentions in his tweets. He did not, however, often reply to tweets; only two out of every 100 messages were replies. Both reply and retweet rates were considerably lower than those characteristic of Trump campaign tweets, which were close to $50 \%$ (Lee \& Lim, 2016, p. 853).

The correlation $(r)$ of each measure with pleasantness is reported in the first column of Table 1 . The single measure most highly correlated with pleasantness was tweet length, with longer tweets being more unpleasant. Hashtags or American flag emojis were found in more pleasant tweets, while mentions of Democrats and fake news were found in more unpleasant ones. As expected, mentions of Melania were associated with more pleasant tweets.

A linear regression with simultaneous entry of all variables was employed to predict pleasantness. Its results are reported in the second column of Table 1 . The prediction as a whole was statistically significant $(p<.001, R=.42)$, and 15 of the 16 predictors were significant contributors to the equation. The only exception was mentions of Mar-a-Lago. According to beta values in Table 1, higher tweet pleasantness was characteristic of messages that included an exclamation mark, were published later in the year, mentioned America, included an American flag emoji, Melania's name, a hashtag, or a mention symbol. Higher unpleasantness (i.e., lower pleasantness) was characteristic of lengthier messages that included mentions of Democrats, fake news, Hillary, CNN, or Fox, and that were retweets or replies.

Correlation and regression analyses confirmed several predictions of the research. Mention of categories such as CNN, fake news, Democrats, and Hillary was associated with and predicted greater unpleasantness. Mention of America or Melania or the inclusion of an American flag emoji was associated with greater tweet pleasantness, but mentions of Mar-a-Lago were not. Unexpectedly, mentions of Fox were not correlated to tweet pleasantness and they entered the regression equation with a significant negative weight, meaning that this variable predicted unpleasantness in a tweet.

\subsection{Popularity}

Liking and retweeting are both measures of tweet popularity. The first indicates that a reader has clicked a button designating her or his approval of the message and the second that the reader has passed the tweet on through their own feed. In the Trump corpus, liking, with a median of 70,714, was more common than retweeting, with a median of $15,805.5$, but liking and retweeting were strongly correlated $(\rho=.88)$ and produced similar results. Both popularity measures were related to pleasantness $(\rho=-.16$ for retweet count, $\rho=-.07$ for likes, $p<.001)$. Because of the overlap between popularity measures, only predictive results for retweeting are reported below.

Table 2 includes the same 16 variables as Table 1 and adds pleasantness to the list. All variables were correlated ( $r$ for pleasantness, length of message, and time of year; and phi for categorical variables) with retweet category. Relationships are reported in the first column of Table 2 . The variable most strongly related to being in the top 50\% of retweets was tweet length-longer tweets were more likely to be retweeted. The variable most strongly related to being in the bottom $50 \%$ was including a mention symbol. The top 50\% retweeted messages also tended to include an exclamation mark, and refer to the topics CNN, Hillary, Democrats, or fake news. Bottom 50\% messages tended to be pleasant retweets or replies that mentioned Fox or included a hashtag. Only four of the 17 variables were totally unrelated to retweet category in the correlation analysis.

A binary logistic regression was performed to predict retweet category (top or bottom 50\%) from the 17 variables. The overall regression was significant $(p<.001)$ and was associated with a Nagelkerke $R$ of .52. It correctly classified $69.5 \%$ of the tweets. The second column of Table 2 reports the odds ratios for each of the variables. The largest odds ratio is 5.34 for mentions of Hillary. This means that if the string Hillary or Clinton appeared in a tweet, it was 5.34 times more likely to fall in the top 50\% 
of retweets. On the other hand if a tweet was a reply, it was only $20 \%$ as likely as a nonreply to fall in the top $50 \%$ of retweets and much more likely to fall in the bottom $50 \%$.

Table2. Results of a Binomial Logistic Regression Predicting Retweet Category (Top or Bottom 50\%) From 17 Tweet Characteristics ${ }^{l}$

\begin{tabular}{|l|c|c|}
\hline & Relationship to retweet category & Odds ratio \\
\hline Tweet characteristics & & $.95^{*}$ \\
\hline Pleasantness & $-.17^{*}$ & $1.05^{*}$ \\
\hline Number of words & $.29^{*}$ & $.95^{*}$ \\
\hline Time of year &. .03 & $.54^{*}$ \\
\hline Is a retweet & $-.26^{*}$ & $.20^{*}$ \\
\hline Is a reply & $-.07^{*}$ & $.74^{*}$ \\
\hline Includes a hashtag & $-.16^{*}$ & $.36^{*}$ \\
\hline Includes a mention & $-.32^{*}$ & 1.10 \\
\hline Tweet content & & .78 \\
\hline Mentions America & -.01 & $5.34^{*}$ \\
\hline Includes American flag emoji & $-.09^{*}$ & 1.26 \\
\hline Mentions Hillary & $.12^{*}$ & $2.59^{*}$ \\
\hline Mentions Democrats & $.10^{*}$ & .66 \\
\hline Mentions fake news & $.14^{*}$ & $4.12^{*}$ \\
\hline Mentions Fox & $-.09^{*}$ & $1.61^{*}$ \\
\hline Mentions CNN & $.08^{*}$ & 1.07 \\
\hline Includes exclamation mark & $.16^{*}$ & .63 \\
\hline Melania & -.04 & .01 \\
\hline Mar-a-Lago & & \\
\hline
\end{tabular}

Note: ${ }^{1}$ Simultaneous entry of all variables, $\mathrm{p}<.001, \mathrm{R}=.52 .{ }^{*} \mathrm{p}<.05$

\section{DISCUSSION}

The prediction that Trump's tweets would be unpleasant was only confirmed in relative terms (relative to Obama's tweets). In absolute terms the tweets were mildly pleasant. Almost all the variables in the research were related to tweet pleasantness (Table 1) and many of them were also related to tweet popularity (Table 2). Predictive schemes (the linear regression predicting pleasantness and the logistic regression predicting popularity) were both significant and successful. Significant contributions of individual variables to prediction generally matched raw correlations, suggesting that there was minimal overlap among predictors. Both tweet characteristics and content variables played a key role in the predictive schemes. Table 3 summarizes the relationships noted in the data by highlighting variables whose presence was related to or predictive of high or low popularity and pleasantness.

Table3. An Overview of Significant Outcomes of Correlation and Regression Analyses

\begin{tabular}{|c|c|}
\hline Celebratory and congratulatory tweets & Antagonistic and accusatory tweets \\
\hline More pleasanttweets are those that ... & More unpleasanttweets are those that ... \\
\hline -are retweeted less often & -are retweeted more often \\
\hline -are liked less often & -are liked more often \\
\hline -include a mention symbol (@) & -mention Democrats \\
\hline -include a hashtag & -mention fake news \\
\hline -include an American Flag emoji & -mention Hillary \\
\hline -were tweeted later in $2017_{p}$ & -mention CNN \\
\hline -mention America & -mention Fox \\
\hline -mention Melania & -are longer \\
\hline -include an exclamation mark & $\begin{array}{l}\text {-are themselves retweets } \\
\text {-are replies } \\
\text { p }_{p}\end{array}$ \\
\hline Less popular tweets are those that ... & More popular tweets are those that ... \\
\hline -are more pleasant & -are more unpleasant \\
\hline -are liked less often & -are liked more often \\
\hline -include a mention (@) & -mention Democrats \\
\hline -include a hashtag & -mention fake news \\
\hline -include an American Flag emoji & -mention Hillary \\
\hline -were tweeted later in $2017_{p}$ & -mention CNN \\
\hline
\end{tabular}




\begin{tabular}{|l|l|}
\hline -are replies & -are longer \\
-are retweets & -include an exclamation mark \\
-mention Fox & \\
\hline
\end{tabular}

Note: ${ }^{1}$ Information from predictive analyses and analyses of relationships was subdivided into categories on the basis of the sign of each correlation or beta weight $(+$ or -$)$ and the size of each odds ratio (below or above 1).

${ }_{p}$ : The subscript ${ }_{p}$ indicates that a variable was only significant as a predictor within a regression equation and was not directly related to the criterion.

Lists in the two right-most cells of Table 3-those describing more unpleasant (top) and more popular (bottom) tweets - are surprisingly similar. Contents and characteristics that point to unpleasant tweets with accusatory or antagonistic language also predict popular ones. Pleasantness and popularity were only weakly correlated $(R=-.17$ for the median split and $\rho=-.16$ for the full range of the retweeting variable), and their overlap seems to be based at least in part on the content variables (mentions of CNN, fake news, Democrats, and Hillary Clinton) and tweet characteristics studied here. Many of the characteristics of unpleasant popular tweets are illustrated in Tweet \#2 (see Measuring the Pleasantness/Unpleasantness of Tweets, above) which was both retweeted $(25,099)$ and liked $(97,362)$ at high rates. Lists in the two left-most cells of Table 3 again show considerable overlap. More pleasant tweets and less popular tweets were similar in many ways. They tended to include mention symbols and hashtags. While popular and unpleasant tweets were antagonistic or accusatory, less popular and pleasant ones tended to be congratulatory or celebratory in nature. Trump posted many such tweets, ranging from congratulatory examples such as Tweet \#1 (see Measuring the Pleasantness /Unpleasantness of Tweets, above)to messages of thanks to various good wishes at Thanksgiving, Christmas, and New Year's. For example, on September 20 Trump tweeted "Thank you @ fox and friends-great show!" This tweet was relatively unpopular (below the median for both likes and retweets) but it was pleasant, with a mean of 79 . The message contained a mention symbol, and was tweeted later in 2017.

Because of the pattern of results reported in Table 3 and the shared characteristics of pleasant / popular tweets on one side and unpleasant / less popular ones on the other, the table's two columns have been provisionally labeled Celebratory and Congratulatory (on the left) and Antagonistic and Accusatory (on the right). These labels may not correctly characterize every single message in the corpus, but they do characterize a majority of them and they point to the presence of two very different kinds of message in the Trump tweet corpus. The 50 most pleasant Trump tweets included the words/phrases Happy (usually Happy Birthday; 15 times), thank you (11), and congratulations (4), as well as the adjective great applied to a performance or activity (7), five messages celebrating Jobs!, two Merry Christmases and six additional celebratory / congratulatory messages of a more general sort. The 50 most unpleasant tweets were less uniform in content, but they tended to include words such as oppose, warning, against, undoing, mishandles, negative, witch hunt, wrong, dirty, fighting, terror, and anti-Trump, and to mention Trump's political opponents.

\subsection{A Post-Hoc Analysis}

Once it became evident that there were two distinct types of messages in the Trump corpus, a question arose as to whether different messages were posted at different times. To answer this question, a single score was created by taking standardized pleasantness and subtracting standardized retweet frequency from it. The top 33\% of messages for this score represented the celebratory/congratulatory category of messages (they were pleasant and less popular) and the bottom 33\% the antagonistic / accusatory one (they were unpleasant and more popular). Frequencies of tweets from the two categories were compared for days of the week and hours of the day.

A $z$ test was employed to compare messages posted on each day of the week. There were only two significant differences: on Mondays, Trump posted preferentially antagonistic/accusatory messages (111 versus 75 messages, $z=2.76, p<.01)$ and on Wednesdays, primarily celebratory/congratulatory ones (158 versus $123, z=2.03, p<.05)$.

When it came to time of day, significant differences were found for two sets of consecutive hours. The beginning of each hour is recorded in the description of results that follows. Celebratory/congratulatory messages predominated for three mid-day hours (11:00 a.m.: 133 versus $67, z=4.60, p<.001 ; 12$ noon: 128 versus $70, z=4.05, p<.001 ; 1: 00$ a.m.: 103 versus $45, z=4.69, p$ 
<.001). Antagonistic/accusatory messages were posted at significantly higher rates later in the day, between 7:00 p.m. and midnight (7:00 p.m.: 46 versus $20, z=3.08, p<.01 ; 8: 00$ p.m.: 52 versus $29, z$ $=2.44, p<.02$; 9:00 p.m.: 53 vs $27, z=2.80, p<.01 ; 11: 00$ p.m.: 62 vs $32, z=2.99, p<.01)$. There was no significant difference for 10:00 p.m. (55 versus $38, z=1.66, p=.10$ ), although the trend continued in the expected direction.

In the cycle of the president's day, late mornings and early afternoons were a preferred time for tweeting emotionally positive messages and the late evenings and nights the preferred time for tweeting emotionally negative messages. Trump's overall tweet frequency was lowest between 4:00 a.m. and 9:00 a.m. and highest between 10:00 a.m. and 1:00 p.m.

\section{CONCLUSIONS}

Donald J. Trump posted both pleasant and unpleasant tweets in 2017, with a small majority of pleasant tweets. More pleasant tweets tended to be congratulatory or celebratory, but not popular. More unpleasant tweets were more popular - they were retweeted more often and liked more often and they tended to be longer and to include mentions of Hillary Clinton, the Democrats, CNN, and fake news. Their tone was antagonistic or accusatory. Celebratory tweets tended to be posted between 11:00 a.m. and 2:00 p.m. and accusatory ones between 7:00 p.m. and midnight.

Political campaigners view negative advertising as advantageous to their cause, and the media tend to highlight and report on negative tactics (Fowler \& Ridout, 2012, p. 60). Unpleasant tweets (which have no upfront cost) could easily be employed in lieu of, or in addition to, negative advertising to create and maintain negative opinions of the opposition among the faithful. According to Ott (2017, p. 60), oversimplified and impulsive tweets make Twitter an ideal medium for shallow, unpleasant, but popular and effective political communications. Superficiality and negativity in political discourse is not limited to the tweetosphere: accusations of creating and maintaining negative opinions of the opposition could also be leveled at liberal-leaning political comedies such as The Daily Show with Trevor Noah.

\section{REFERENCES}

[1] Borowitz, A. (2018). Satire from the Borowitz Report: Trump furious after Twitter's bot purge leaves him with fourteen followers. The New Yorker, February 21. Retrieved from https://www.newyorker.com/ humor/borowitz-report/trump-furious-after-twitters-bot-purge-leaves-him-with-fourteen-followers

[2] Brown, B. (2018). Up-to-date archive of Trump tweets. Retrieved from https://github.com/bpb27/trump_ tweet_data_archive

[3] Enli, G. (2017). Twitter as arena for the authentic outsider: Exploring the social media campaigns of Trump and Clinton in the 2016 U.S. presidential election. European Journal of Education, 32, 50-61.

[4] Fowler, E., \& Ridout, T. (2012). Negative, angry, and ubiquitous: Political advertising in 2012. Forum, 10, $51-61$.

[5] Gravetter, F., Wellnau, B., \& Forzano, L. (2018). Essentials of statistics for the behavioral sciences (9th ed.) Boston, MA: Cengage Learning.

[6] The Internet Archive. (n.d.). POTUS twitter archive zip. Retrieved from https://archive.org/details/Obama White House Pot us Twitter Data

[7] Lee, J., \& Lim, Y. (2016). Gendered campaign tweets: The cases of Hillary Clinton and Donald Trump. Public Relations Review, 42, 849-855.

[8] Mohammad, S. M., Kiritchenko, S., \& Zhu, X. (2013, August 28). NRC-Canada: Building the state-of-theart in sentiment analysis of tweets. Retrieved from arXiv:1308.6242vl [cs.CL]

[9] Ott, B. (2017). The age of Twitter: Donald J. Trump and the politics of debasement. Critical Studies in Media Communication, 34, 59-68.

[10] Saif, H., Fernandez, M., He, Y, \& Alani, H. (2016). Evaluation datasets for Twitter sentiment analysis. Paper presented at the first workshop on emotion and sentiment in social and expressive media (ESSEM), in conjunction with AI*IA conference, Turin, Italy. Retrieved from ceur-ws.org/Vol-1096/paper1.pdf

[11] Wang, Y., Luo, J., Niemi, R., Li, Y., \& Hu, T. (2016). Catching fire vialikes: Inferring topic preferences of Trump followers on Twitter. Proceedings of the Tenth International AAAI Conference on Web and Social Media (ICWSM). Retrieved from arXiv:1603.03099[cs.SI]

[12] Whissell, C. (2009). Using the revised Dictionary of Affect in Language to quantify the emotional undertones of samples of natural language. Psychological Reports, 105, 509-521. 
[13] Whissell, C. (2010a). Emotion and the humors: Scoring and classifying major characters from Shakespeare's comedies on the basis of their language. Psychological Reports, 106, 813-831.

[14] Whissell, C. (2010b). Leading with words? Emotion and style in the language of U.S. president Clinton's public communications. Psychology of Language and Communication, 14, 81-91.

Citation: Cynthia Whissell. "Trump Tweets". International Journal on Studies in English Language and Literature (IJSELL), vol 6, no.10, October2018, pp. 32-40. doi:http://dx.doi.org/10.20431/23473134.0610004.

Copyright: (C) 2018 Authors. This is an open-access article distributed under the terms of the Creative Commons Attribution License, which permits unrestricted use, distribution, and reproduction in any medium, 